\title{
A short remark on the modelling of a three-story shear-building structure
}

\author{
Marcus Varanis ${ }^{* 10}$, Anderson Langone Silva $^{1}$, Arthur Guilherme Mereles ${ }^{1}$ \\ ${ }^{1}$ Universidade Federal da Grande Dourados, Faculdade de Engenharia, Rodovia Dourados - Itahum, s/n, Cidade Universitária, \\ Dourados, MS, Brasil
}

Received on April 25, 2018; Revised on June 27, 2018; Accepted on July 19, 2018.

\begin{abstract}
In this note, some remarks on the modelling of a multi-degree-of-freedom system, a three-story shear-building structure, is presented. The modelling of a mechanical system is one of the most important aspects in engineering and physics, since is crucial for the understanding of the system. Besides, the model, if properly made, can be also useful to predict some phenomenon that might appear on the system itself and is unknown to researchers. In many situations, the system studied is simplified as a physical model, where the main characteristics regarding the dynamics of the system are defined, such as inertia and stiffness. This facilitates the obtainment of the mathematical model, which is used to obtain quantitative results that can be checked in some experimental procedure. This work presents the modeling of a shear-building structure, where the equations of motion and the natural frequencies of oscillation are obtained. The numeric values are validated by the comparison with experimental results from a previous work.
\end{abstract}

Keywords: multi-degree-of-freedom systems, mechanical vibration, mathematical modelling, shear-building structure

\section{Introduction}

It is very important in the study of a real system the modelling of it, which translates its components and effects into a more common ground, where they can be more closely studied. This common ground is the physical theories and through them the researchers can make physical models, which represent the real system at some extent. The physical models are made by representing some aspects of the system that can be quantified and used to predict the possible future effects that may occur in it. The quantitative results obtained from the model can be validated by an experiment procedure, which proves the suitability of the model in representing the real system.

In this note, it is presented the modelling of a threestory shear-building structure, where the equations that govern the system are obtained. To validate the model, the natural frequencies of the structure are analytically obtained and compared to experimental data from a previous work [1]

\section{Shear-building model}

The shear-building structure consist in one of more rigid masses supported by flexible columns that are subjected to shear stresses as the masses oscillate laterally. This model is used very often in the design of buildings mainly

*Correspondence email address: marcusvaranis@ufgd.edu.br to estimate the natural frequencies of them. The shearbuilding studied in this work is shown in Figure 1.a) and consist of three rigid masses connected by flexible columns. The masses are allowed to oscillate only laterally. Also, an unbalanced electric motor is mounted on top of the structure to provide a harmonic excitation.

Since the main objective is to obtain the natural frequencies of the shear-building structure, the system was modelled as lumped masses connected by springs and dampers, as shown in Figure 1.b). This approach facilitates the analysis of the system and gives accurate results if the masses are rigid and the columns flexible enough. The model has three degrees-of-freedom (DOF), since three coordinates are needed to describe the movement of the three masses.

The equivalent spring stiffness of the structure's columns is obtained using Equation (1) [1-2]; where $E$ denotes the Young's modulus of the column's material, $l$ is the the column's length and $I$ is the moment of inertia of the cross-sectional area, which, for a rectangular crosssectional area, is given by Equation (2), being $b$ and $h$ the column's width and thickness, respectively

$$
\begin{gathered}
k=\frac{3 E I}{l^{3}} \\
I=\frac{b h^{3}}{12}
\end{gathered}
$$

The equations of movement of the system (EOM) can be obtained by applying the Newton's second law in each 


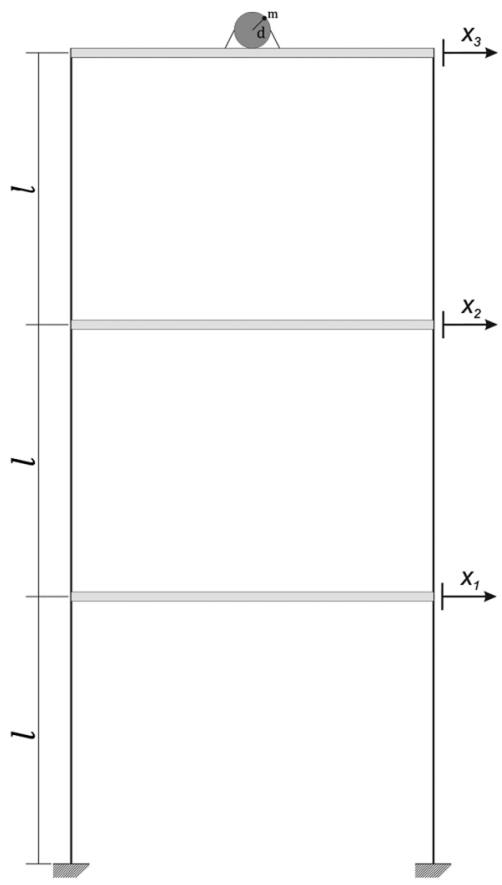

a)

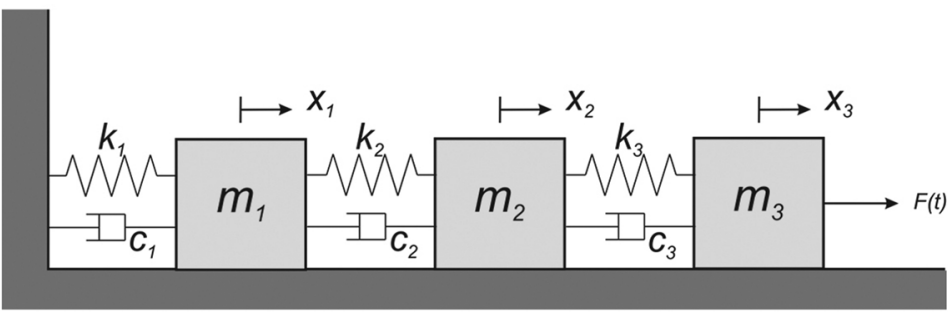

b)

Figure 1: (a) Three-story shear-building and (b) equivalent spring-mas system (Taken from [1]).

mass, giving the following results

$$
\begin{aligned}
& {\left[\begin{array}{ccc}
m_{1} & 0 & 0 \\
0 & m_{2} & 0 \\
0 & 0 & m_{3}
\end{array}\right]\left\{\begin{array}{l}
\ddot{x}_{1} \\
\ddot{x_{2}} \\
\ddot{x_{3}}
\end{array}\right\}} \\
& +\left[\begin{array}{ccc}
c_{1}+c_{2} & -c_{2} & 0 \\
-c_{2} & c_{2}+c_{3} & -c_{3} \\
0 & -c_{3} & c_{3}
\end{array}\right]\left\{\begin{array}{l}
\dot{x_{1}} \\
\dot{x_{2}} \\
\dot{x_{3}}
\end{array}\right\} \\
& +\left[\begin{array}{ccc}
k_{1}+k_{2} & -k_{2} & 0 \\
-k_{2} & k_{2}+k_{3} & -k_{3} \\
0 & -k_{3} & k_{3}
\end{array}\right]\left\{\begin{array}{l}
x_{1} \\
x_{2} \\
x_{3}
\end{array}\right\} \\
& =\left\{\begin{array}{c}
0 \\
0 \\
F(t)
\end{array}\right\} \begin{array}{c} 
\\
{[M]\{\ddot{x}\}+[C]\{\dot{x}\}+[K]\{x\}=[F]}
\end{array}
\end{aligned}
$$

Where $[M],[C]$ and $[K]$ are the mass, damping and stiffness matrices, respectively, $\{x\}$ is the displacement vector of the system, $[F]$ is the external force vector applied in the system and the dots represent time derivatives. It is worth noting that the top mass of the structure, $m_{3}$, has a higher value than the other masses due to the electrical motor positioned on top of it. To simplify the analysis, the damping of the structure is considered to be zero, which is a reasonable approach for a light-damped structure. Thus Equation (4) can be written as,

$$
[M]\{\ddot{x}\}+[K]\{x\}=[F]
$$

The solution of the free vibration of Equation (5), that is when $[F]=0$, is given by,

$$
\{x(t)\}=\{\phi\} e^{j \omega_{n} t}
$$

Being $\phi$ the modes of vibration vector corresponding to the natural frequency $\omega_{n}$ By substituting Equation (6) into $(5)$, with $[F]=0$, one may have,

$$
\left[[K]-\omega_{n}^{2}[M]\right] \phi=0
$$

From Equation (7), the following characteristic equation is obtained,

$$
\operatorname{det}\left(\left[[K]-\omega_{n}^{2}[M]\right]\right)=0
$$

Equation (8) is a third order polynomial equations which solution gives the natural frequencies of the system, $\omega_{n 1}$, $\omega_{n 2}$ and $\omega_{n 3}$. The modes of vibration are obtained by substituting the natural frequencies in Equation (7). The mass and stiffness matrices used to obtain the natural frequencies are given by the following

$$
\begin{aligned}
& {[K]=\left[\begin{array}{ccc}
3.932 & -1.966 & 0 \\
-1.966 & 3.932 & -1.966 \\
0 & -1.966 & 1.966
\end{array}\right](\mathrm{kN} / \mathrm{m})(9)} \\
& {[M]=\left[\begin{array}{ccc}
0.416 & 0 & 0 \\
0 & 0.416 & 0 \\
0 & 0 & 0.726
\end{array}\right](\mathrm{kg})(10)}
\end{aligned}
$$

Where the mass of the third floor of the structure is greater because it accounts the mass of the unbalanced motor. Applying these matrices in equation (8), which is a characteristic equation for an eigenvalue and eigenvector 
problem, the first, second and third natural frequencies are obtained analytically, which are 4.09, 12.54 and 19.32 $\mathrm{Hz}$, respectively. These results are in agreement with the analytical and experimental values shown in Ref. [1]. It is worth noting that the mass and stiffness matrices presented in Ref. [1] were written incorrectly, as the mass of the third floor does not account the electric motor, but the results of that work were correct as it is presented here

\section{Conclusions}

This note presented some remarks on the modeling of a multi-DOF system consisting of a shear-bulding structure with three stories. The structure was modeled as a spring-mass system with each mass corresponding to the structure story. The damping was not considered in the model as the system was ligh-damped. with experimental values The natural frequencies obtained from the model were in agreement from a previous work [1], which presented incorrect mass $([M])$ and stiffness $([K])$ matrices, since the mass of the electric motor was not added in the former.

\section{References}

[1] M. Varanis, A. Silva and A. Mereles, Rev. Bras. Ens. Fis., 40, e1304 (2018).

[2] S.S. Rao and F.F. Yap, Mechanical Vibrations (AddisonWesley, Boston, 1995), $3^{\mathrm{a}}$ ed. 